\title{
Application of Dual-Frequency Self-Injection Locked DFB Laser for Brillouin Optical Time Domain Analysis
}

\author{
Cesar A. Lopez-Mercado ${ }^{1}$, Dmitry A. Korobko ${ }^{2}$, Igor O. Zolotovskii ${ }^{2}$ and Andrei A. Fotiadi ${ }^{2,3,4, *(D)}$ \\ 1 Scientific Research and Advanced Studies Center of Ensenada (CICESE), 22860 Ensenada, BC, Mexico; \\ cmercado@cicese.edu.mx \\ 2 S.P. Kapitsa Research Institute of Technology, Ulyanovsk State University, 42 Leo Tolstoy Street, \\ 432970 Ulyanovsk, Russia; korobkotam@rambler.ru (D.A.K.); rafzol.14@mail.ru (I.O.Z.) \\ 3 Electromagnetism and Telecommunication Department, University of Mons, B-7000 Mons, Belgium \\ 4 Ioffe Physical-Technical Institute of the RAS, 26 Polytekhnicheskaya Street, 194021 St. Petersburg, Russia \\ * Correspondence: Andrei.FOTIADI@umons.ac.be
}

Citation: Lopez-Mercado, C.A.; Korobko, D.A.; Zolotovskii, I.O.; Fotiadi, A.A. Application of Dual-Frequency Self-Injection Locked DFB Laser for Brillouin Optical Time Domain Analysis. Sensors 2021, 21, 6859. https://doi.org/10.3390/ s21206859

Academic Editor: Maria Konstantaki

Received: 31 August 2021

Accepted: 12 October 2021

Published: 15 October 2021

Publisher's Note: MDPI stays neutral with regard to jurisdictional claims in published maps and institutional affiliations.

Copyright: (c) 2021 by the authors. Licensee MDPI, Basel, Switzerland. This article is an open access article distributed under the terms and conditions of the Creative Commons Attribution (CC BY) license (https:// creativecommons.org/licenses/by/ $4.0 /)$.

\begin{abstract}
Self-injection locking to an external fiber cavity is an efficient technique enabling drastic linewidth narrowing of semiconductor lasers. Recently, we constructed a simple dual-frequency laser source that employs self-injection locking of a DFB laser in the external ring fiber cavity and Brillouin lasing in the same cavity. The laser performance characteristics are on the level of the laser modules commonly used with BOTDA. The use of a laser source operating two frequencies strongly locked through the Brillouin resonance simplifies the BOTDA system, avoiding the use of a broadband electrooptical modulator (EOM) and high-frequency electronics. Here, in a direct comparison with the commercial BOTDA, we explore the capacity of our low-cost solution for BOTDA sensing, demonstrating distributed measurements of the Brillouin frequency shift in a $10 \mathrm{~km}$ sensing fiber with a $1.5 \mathrm{~m}$ spatial resolution.
\end{abstract}

Keywords: distributed fiber sensing; BOTDA; Brillouin fiber laser

\section{Introduction}

Distributed optical fiber sensors show superior advantages over their electronic counterparts due to their high sensitivity to external disturbances and low loss transmission, which are important for remote sensing [1]. Unlike single-point optical fiber sensors [2,3], distributed optical fiber sensors can interrogate and spatially resolve measurands along an optical fiber due to their specific sensing mechanisms [4-7]. Among them, Brillouin-based distributed sensors [8-10] have attracted immense interest in recent years in fields such as the health monitoring of large structures in oil and gas pipelines [11], railways and highvoltage transmission lines [12], high-temperature distributed measurement in industrial applications [13], distributed strain measurement for cracks detection [14], and structural health monitoring [15]. The conventional distributed Brillouin optical fiber sensing is based on the backward-stimulated Brillouin scattering, where the strain or temperature is a linear function of the Brillouin frequency shift (BFS) and so can be recovered from the distribution of Brillouin gain spectra (BGS) along the sensing fiber [16,17]. Over the past two decades, many efforts have been devoted to improving its performance, including spatial resolution, measurement time, and sensing range [18-20]. Although the Brillouin sensing instruments have become commercially available, their relatively high cost remains the major critical factor limiting the range of their potential applications. A key and most expensive part of the traditional BOTDA system is a master-oscillator module employed for generation and tuning of the pump and Stokes signal frequencies. Commonly, such modules implement phase-locked loop (PLL) [21] or optical side-band (OSB) generation techniques [22]. In the PLL technique, two narrowband laser sources are used to generate pump and Stokes laser frequencies. The frequency of one laser is locked to the frequency of another laser through 
a feedback circuit, which allows tuning the frequency difference. This technique requires the use of high-frequency photodetectors and RF generators. In the OSB method, only one narrowband laser is used, whereas the second frequency is generated through a broadband electro-optical modulator (EOM) driven at the frequency corresponding to the desired frequency shift $(\sim 11 \mathrm{GHz}$ ). This technique employs a broadband EOM and high-frequency drivers. Therefore, besides the narrowband laser sources with strict stability requirements, both methods require the use of rather costly high-frequency devices and electronic circuits. Considerable efforts have been directed to simplifying the generation and tuning of pump and Stokes signals. A number of new BOTDA solutions have been proposed. Among them are sensors employing a single optical source driven by pulsed RF signals and passive optical filtering [23], systems based on time-division pump-probe generation by direct modulation of a laser diode through an arbitrary waveform generator [24], and sources that use the Stokes wave generated inside a highly nonlinear fiber [25]. The idea to use a Brillouin fiber laser has been raised and investigated for this purpose [26-28]. However, stable lasers are supplied by rather expensive active stabilization circuits. Concerning the capacity of BOTDA systems based on Brillouin laser sources, the previous works were not conclusive and no comparison with the commercial BOTDA systems was performed.

An alternative solution for a dual-frequency laser potentially suitable for BOTDA applications was reported recently [29]. The principle of the laser operation is based on the self-injection locking mechanism, which enables the reduction in the DFB laser linewidth down to the sub-kilohertz level [30]. The ability of this low-cost source to replace the standard laser in the phase-OTDR analyzer has been experimentally demonstrated [31,32]. In the dual-frequency laser configuration [33], the same ring fiber cavity is used to generate narrow-band coherent light at the pump frequency (through the self-injection locking mechanism) and narrow-band coherent light at Stokes frequency (through stimulated Brillouin scattering). The laser is supplied with a simple low-bandwidth active optoelectronic feedback circuit governed by a low-cost USB-DAQ card [34]. Importantly, the drastic narrowing of the DFB laser linewidth to the sub-kilohertz range is provided by the selfinjection-locking mechanism, whereas the active feedback is used only to maintain the laser operation in this regime. Therefore, in terms of feedback circuit bandwidth, complexity, and allocated memory, this method is much less consuming than the optoelectronic systems commonly used with Brillouin fiber lasers, including the lasers earlier considered for BOTDA sensing [27].

In this paper, we explore the potential of this low-cost laser solution for operation with the Brillouin optical time domain analyzer (BOTDA) [17]. We present an extended description of the experimental results, evaluating the capacity of the reported laser configuration for distributed measurements of the Brillouin frequency shift (BFS) in a $10 \mathrm{~km}$ fiber testing line with a spatial resolution of $1.5 \mathrm{~m}$. The control measurements in the same testing line were performed with a commercial BOTDA set (OZ-optics, OZ Optics Limited, Canada) for investigation using the same spatial resolution. A direct comparison of the results highlights no deterioration in the BOTDA system characteristics associated with the use of low-cost dual-frequency laser. Importantly, the laser source operating at two frequencies strongly locked through the Brillouin resonance simplifies the BOTDA system and excludes the use of a broadband EOM and the high-frequency electronics commonly employed with the BOTDA system based on a single master-oscillator.

\section{Experimental Setup}

Our experimental BOTDA configuration is shown in Figure 1. A simple self-injection locked Brillouin laser operating at $1535 \mathrm{~nm}$ [33] was used as a master-oscillator. The laser generated two monochromatic optical waves at pump $\left(v_{L 0}\right)$ and Stokes $\left(v_{S 0}\right)$ frequencies delivered through two independent fiber outputs. The laser operation is characterized by natural Lorenz linewidths of $\sim 270$ and $\sim 110 \mathrm{~Hz}$, and powers of $\sim 9 \mathrm{~mW}$ and $\sim 100 \mu \mathrm{W}$ for pump and Stokes outputs, respectively. The laser optical spectra shown in Figure 2 demonstrate the contrast of $\sim 70 \mathrm{~dB}$ between the peak and background powers for both laser 
outputs; the spectra are centered at 1531.13 and $1531.21 \mathrm{~nm}$, respectively. The spectrum widths are determined by the resolution $(\sim 0.01 \mathrm{~nm})$ of the spectrum analyzer. The difference of $\sim 0.08 \mathrm{~nm}$ between the peak wavelengths corresponds to a Brillouin frequency shift (BFS) of $\sim 11 \mathrm{GHz}$. A weak Rayleigh scattering signal at the pump frequency was also observed through the Stokes laser output.

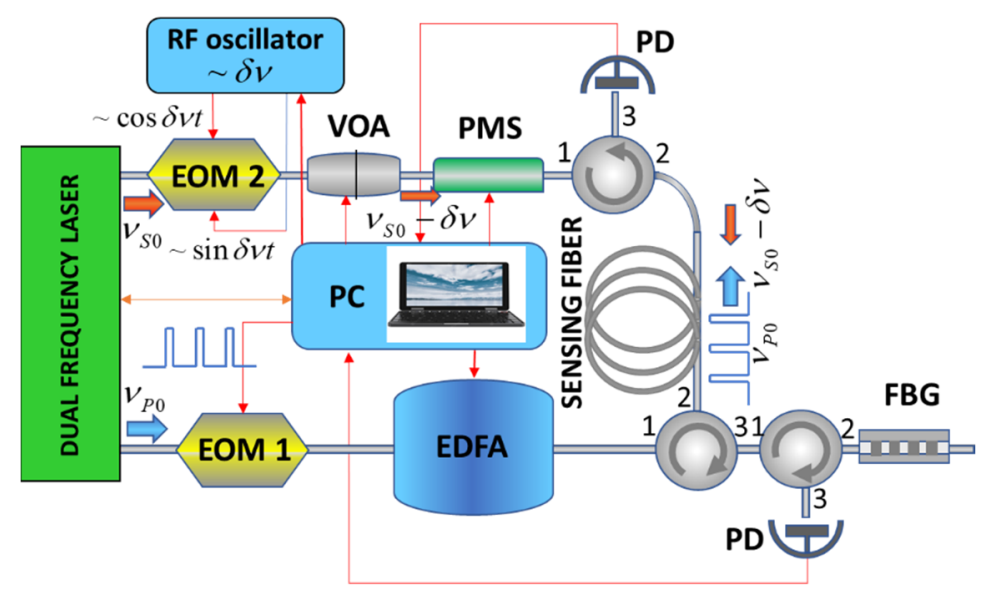

Figure 1. The experimental BOTDA setup.

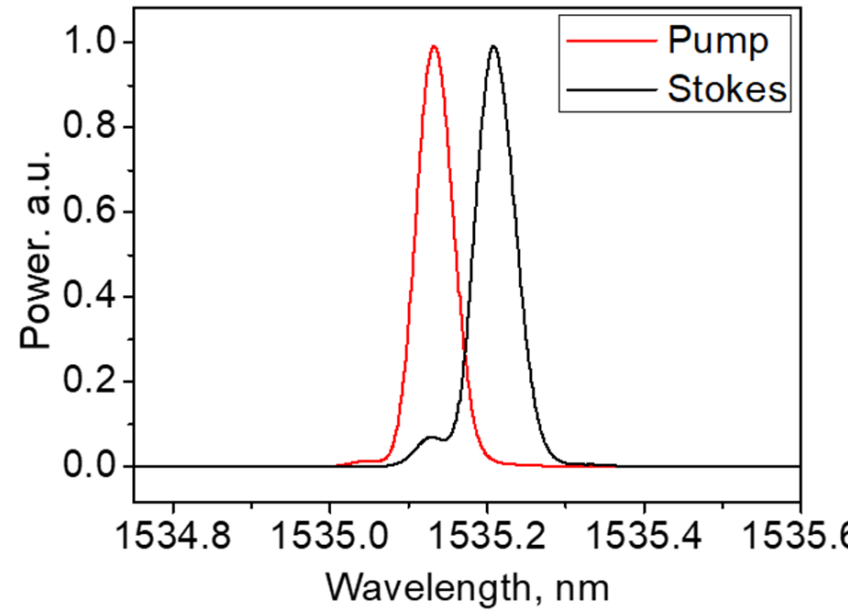

(a)

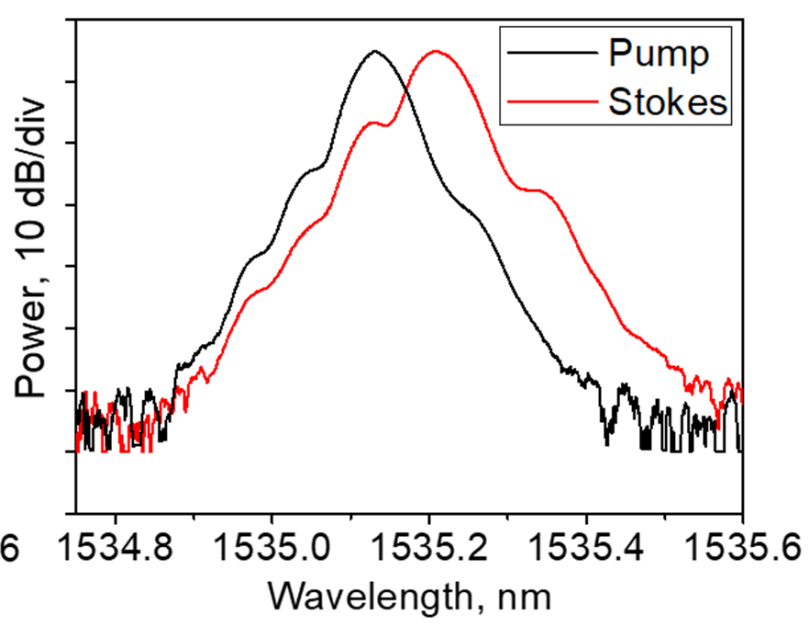

(b)

Figure 2. Optical spectra recorded at two laser outputs in (a) linear and (b) decibel scales.

The beating between two laser outputs is characterized by a stable radio-frequency (RF) spectrum with $290 \mathrm{~Hz}$-linewidth and fixed peak frequency at $v_{L 0}-v_{S 0}=\Delta v_{0} \sim$ 10.946 GHz, as presented in Figure 3a. The peak RF spectrum corresponds to the Brillouin frequency shift in SMF-28 fiber (Corning, Inc., Corning, NY, USA) used in the laser cavity at $1535 \mathrm{~nm}$. To reduce the effect of the environmental noise, a spliced laser configuration was placed into a thermostabilized $\left(\sim 25^{\circ} \mathrm{C}\right)$ foam box. Additional thermal control was applied to the laser box as a whole and used to keep the feedback circuit within its dynamic range ensuring long-term laser operation stability. The drift in the RF beat recorded each minute for $1 \mathrm{~h}$ is shown in Figure $3 \mathrm{~b}$. Variations in the RF spectrum peak frequency within a limited range $\left(\delta v_{R F}<5 \mathrm{kHz}\right)$ reflects the effect of the thermal control. No mode hopping event was observed when the RF beat frequency was within this range. Details on the laser operation and its performance characteristics are provided in Appendix A. 


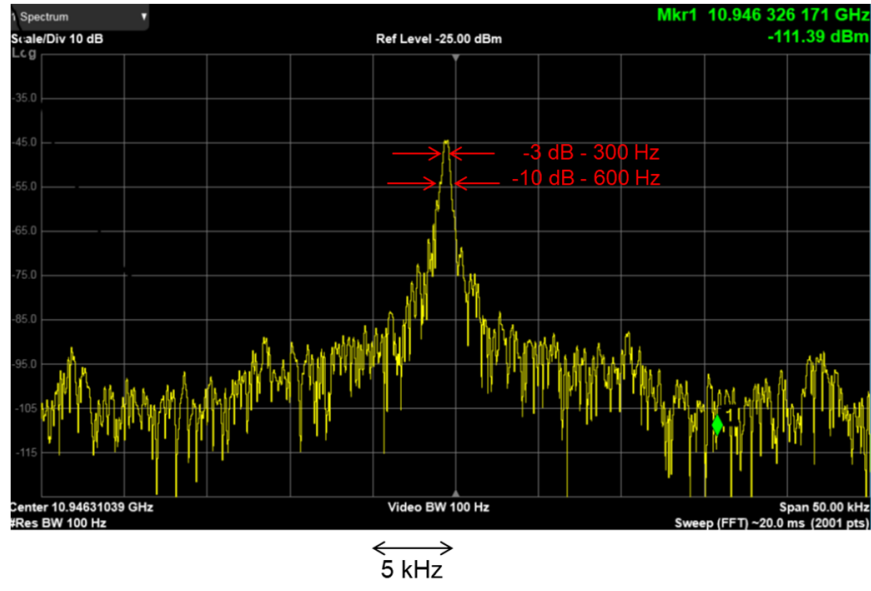

(a)

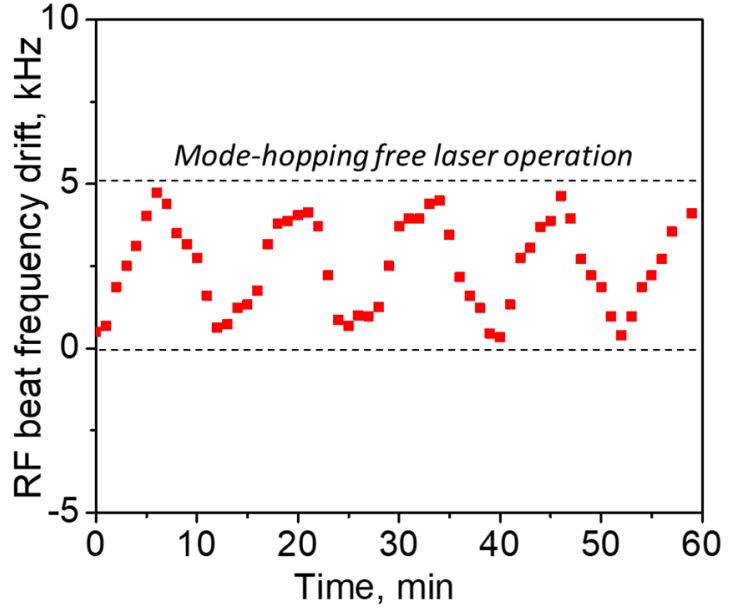

(b)

Figure 3. (a) Typical RF beat note spectrum measured with two master-oscillator outputs; (b) drift in the RF beat frequency measured each minute for $60 \mathrm{~min}$.

The BOTDA built in the laboratory is a simple modification of the traditional pumpprobe setup [35]. The laser radiation at the pump frequency $v_{L 0}$ passes through the electro-optical intensity modulator (EOM 1) and erbium-doped fiber amplifier (EDFA) to form a periodic train of rectangular pulses with the peak power of $\sim 300 \mathrm{~mW}$, pulse duration of $\sim 15 \mathrm{~ns}$, and repetition rate of $\sim 10 \mathrm{kHz}$. The laser radiation emitted at the Stokes frequency $v_{S 0}$ passes through the dual-drive electro-optic modulator (EOM 2, SSB-CS EOM, Sumitomo), variable optical attenuator (VOA), and polarization mode scrambler (PMS) to form a $\sim 10 \mu \mathrm{W} \mathrm{CW}$ probe signal at the frequency $v_{S}=v_{S 0}-\delta v_{S}$. The frequency tuning of the probe signal is provided by a tunable radio-frequency generator supplying EOM 2 at $\delta v_{S}<1 \mathrm{GHz}$. The pump pulses at $v_{L 0}$ and the $\mathrm{CW}$ probe signal at $v_{S}$ were introduced into the fiber under test from the opposite fiber ends. Their interaction through the Brillouin process in the fiber under test caused an energy transfer from the pump pulse to the CW Stokes signal, leading to its intensity modulation recorded at the fiber output by the fast photodetector and PC acquisition card. The modulation amplitude (typically <100 nW) of the probe signal is proportional to the local Brillouin gain at the fiber point where Brillouin resonance is achieved. The recorded probe signal traces were averaged over 4096 pump pulses and used to map the distribution of the Brillouin gain over the fiber length. The traces recorded at different $\delta v_{S}$ were used to build the Brillouin gain spectrum at each fiber point. Then, these data were mathematically processed to find the position of the Brillouin spectrum peak, i.e., the BFS, in each fiber point. The pump pulse duration of $\sim 15$ ns sets the system spatial resolution of $\sim 1.5 \mathrm{~m}$. Notably, the use of the laser source operating two frequencies strongly locked through the Brillouin resonance as a master-oscillator in our BOTDA setup allowed replacing the broadband EOM used in [35] for the frequency shift by $\sim 11 \mathrm{GHz}$ and all high-frequency electronics by more cost-efficient and straightforward counterparts operating in the sub-gigahertz radio-frequency range.

To evaluate the performance of the laser operation with the built BOTDA, we built a fiber testing line similar to that commonly used with BOTDAs for their calibration. The $10 \mathrm{~km}$ length testing line shown in Figure 4 comprises two lengths of SMF-28 Corning fiber (9.1 and $1 \mathrm{~km})$ and a length of OFS (G.657) fiber $(\sim 50 \mathrm{~m})$ placed between them. The latter includes eleven altered fiber coils of different lengths $(0.75-6.0 \mathrm{~m})$ kept at different temperatures. Odd coils were placed into a heat chamber thermostabilized at $60{ }^{\circ} \mathrm{C}$, while even coils were rested at room temperature $\left(\sim 25^{\circ} \mathrm{C}\right)$. The measured distribution of the BFS over the OFS fiber followed the temperature fiber profile with a factor of $\sim 1 \mathrm{MHz} /{ }^{\circ} \mathrm{C}$. 


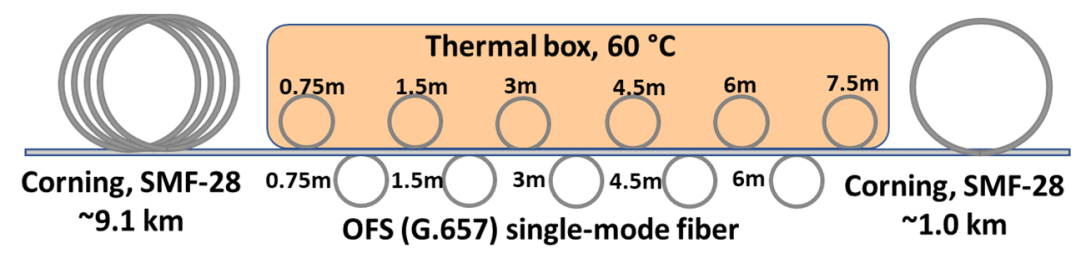

Figure 4. Optical fiber line for BOTDA testing.

\section{Results}

The testing experiments were performed with our BOTDA setup and then repeated with the commercial BOTDA system (OZ-optics) set to operate with a pump pulse of $15 \mathrm{~ns}$. Note that the operating wavelength of the commercial BOTDA is $1550 \mathrm{~nm}$, so the BFS data measured with this device are presented here with a scaling factor of 1550/1535. The experimental results are shown in Figures 5 and 6. First, we adjusted the frequency difference between the pump and probe signal $\Delta v_{0}+\delta v_{S}$ to maximize the signal recorded from the first length of the SMS-28 fiber. In this case, $\delta v_{S} \approx 0$, since the frequency difference of the two signals emitted by the laser corresponds to the BFS in the SMS-28 fiber at room temperature $(\sim 10.946 \mathrm{GHz})$. Figure 5a compares the traces recorded with our setup and the commercial BOTDA: both traces exhibit similar behavior, including the gain modulation pronounced in the fiber points with an alternating temperature. The drop in both traces after $9.2 \mathrm{~km}$ is explained by pump power loss after splicing. Figure $5 \mathrm{~b}$ provides more details on the Brillouin gain distribution along the OFS fiber. Both systems well-recognized the fiber segments where the fiber temperature was higher than the room temperature. Since the BFS increases with temperature, the BFS in hot OFS fiber segments is closer to the frequency difference set by the system, leading to an increase in the local Brillouin gain measured at these points.

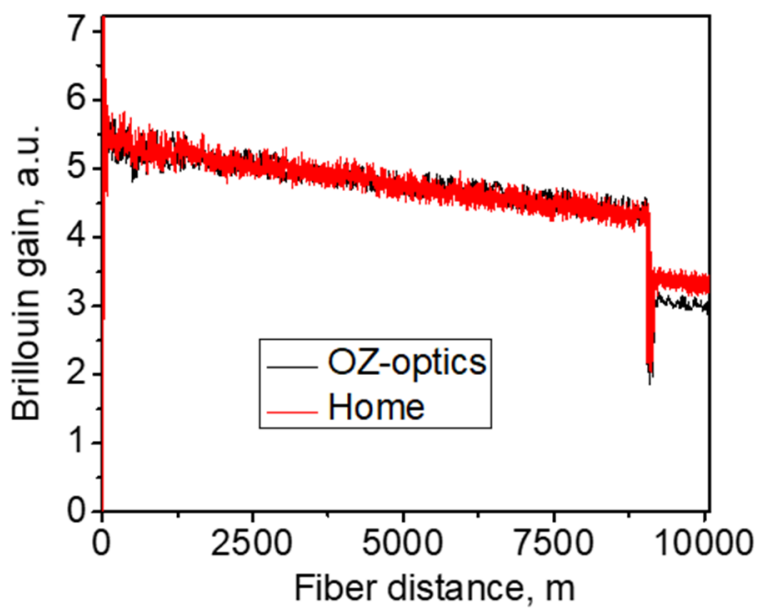

(a)

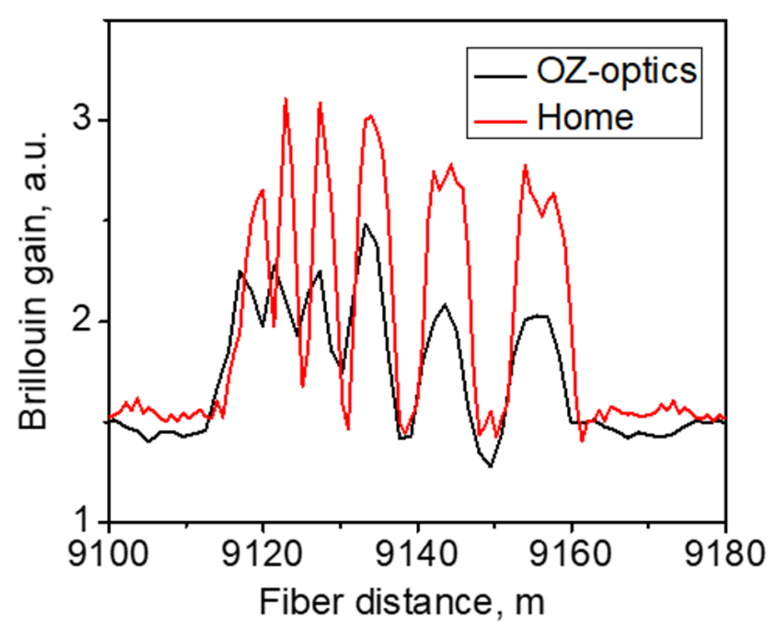

(b)

Figure 5. The measured distribution of the Brillouin gain (a) over the whole testing line and (b) over the range of 9.1-9.18 km.

In order to accurately estimate the BFS distribution along the sensing fiber, the Brillouin gain spectrum (BGS) was measured at each fiber location. At each fiber point, the radio frequency $\delta v_{S}$ applied to the dual-drive EOM 2 was swept within the range of 100 $\mathrm{MHz}$ with a step of $\sim 2 \mathrm{MHz}$. Then, the BFS was reconstructed by fitting the recorded data with the Lorentzian profile [17]. Figure 6a compares the BGSs at two OFS fiber points located at 9151 and $9057 \mathrm{~m}$ measured with our setup and the commercial BOTDA. Our system well-reproduced the BGS shape recorded with the commercial BOTDA. Notably, the BGS corresponding to the fiber points exposed to the temperature $\sim 25$ and $\sim 60{ }^{\circ} \mathrm{C}$ are distant by $35 \mathrm{MHz}$. Figure 6b shows the distributions of the BFS over the OFS fiber: the quality of BSF restoration is rather high. For the fiber segments longer than $1.5 \mathrm{~m}$, both devices produced almost the same distribution, providing a good agreement in absolute 
peak frequencies varying from $10,880 \mathrm{GHz}$ (at $\sim 25^{\circ} \mathrm{C}$ ) to $10,915 \mathrm{GHz}$ (at $\sim 60^{\circ} \mathrm{C}$ ), resolved slopes, and segment positions. All specific fiber segments were well-recognized. For the fiber segment shorter than $1.5 \mathrm{~m}$, our experimental device demonstrated even higher accuracy than the commercial BOTDA operating with the same pulse duration.

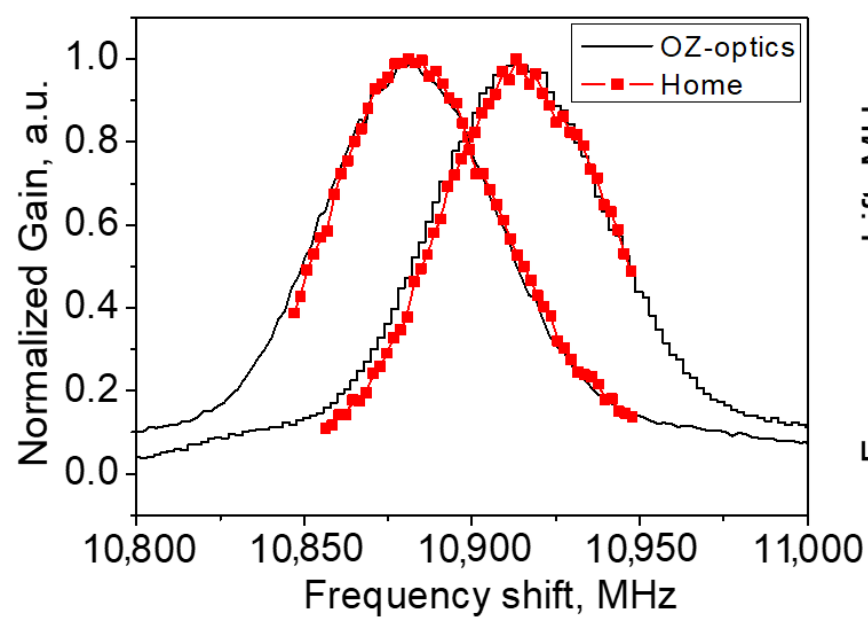

(a)

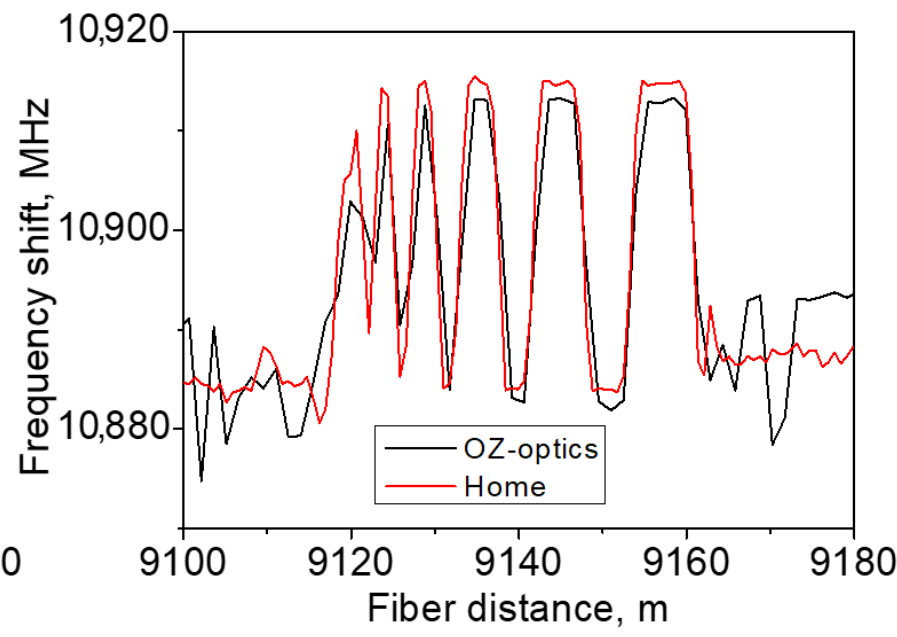

(b)

Figure 6. (a) The Brillouin gain spectra (BGS) measured at the fiber points of 9151 and $9157 \mathrm{~m}$ exposed to the temperature of $\sim 25$ and $\sim 60^{\circ} \mathrm{C}$, respectively; (b) the measured distribution of the Brillouin frequency shift (BFS) over the range of 9.1 to $9.18 \mathrm{~km}$.

\section{Conclusions}

In conclusion, we used a simple dual-frequency laser configuration operated with BOTDA. The use of the laser operating two fixed frequencies significantly simplified the BOTDA system and allows excluding the broadband EOM (and high-power high-frequency microwave generator supplying the EOM) from the traditional BOTDA configuration based on a single narrowband laser source. These relatively high-cost devices constitute a significant part of the BOTDA system value. Additionally, the laser operation mechanism enabling self-stabilized generation of the Brillouin wave does not employ rather an expensive wavelength locking circuit, being a part of the Brillouin lasers offered for BOTDA sensing earlier [27]. Instead, a dual-drive EOM driven by a standard $1 \mathrm{GHz}$ radio-frequency generator is employed in our home BOTDA setup. Moreover, the tuning range of $100 \mathrm{MHz}$ was enough to perform the measurements shown in Figure 6. Under control of the commercial BOTDA, we evaluated the capacity of a low-cost laser solution to operate with the BOTDA sensing, demonstrating the distributed measurements of the BFS in a $10 \mathrm{~km}$ sensing fiber with a $1.5 \mathrm{~m}$ spatial resolution. No deterioration of the system performance characteristics associated with the use of the dual-frequency laser was found during the measurements. Further research will be directed to design and testing of new laser sources [36] for advanced sensor applications.

Author Contributions: Conceptualization, A.A.F.; methodology, A.A.F.; software, I.O.Z.; validation, C.A.L.-M., D.A.K. and I.O.Z.; formal analysis, D.A.K.; investigation, C.A.L.-M.; resources, A.A.F.; data curation, D.A.K.; writing—original draft preparation, A.A.F.; writing—review and editing, A.A.F.; visualization, C.A.L.-M.; supervision, A.A.F.; project administration, A.A.F.; funding acquisition, A.A.F. All authors have read and agreed to the published version of the manuscript.

Funding: This research was funded By the Ministry of Science and Higher Education of the Russian Federation, grant number 075-15-2021-581 and the Russian Science Foundation, grant number 18-1200457P. 
Data Availability Statement: Not applicable.

Conflicts of Interest: The authors declare no conflict of interest.

\section{Appendix A. Dual-Frequency Self-Injection-Locked DFB Laser}

This section contains details and supplementary data on the low-cost dual-frequency master-oscillator described earlier in [33] and used in these studies with the BOTDA setup shown in Figure 1. A simple laser configuration was spliced from standard telecom components. It employed a single DFB laser coupled to an all-fiber ring cavity. In this configuration, the same ring fiber cavity is used both for self-injection locking of the DFB laser and for generation of Stokes light through the Brillouin lasing. A low-cost USB-DAQ was used to stabilize the system, preventing mode-hopping. Specifically, a narrowband laser operation was provided by the self-injection locking mechanism, whereas the active feedback circuit just helped the laser maintain the desired operation. Additionally, the selfinjection locking supported permanent coupling between the DFB laser and the external fiber ring cavity, enabling an efficient resonant pumping for low-noise Brillouin wave generation. The ability to emit light from a single DFB laser at two frequencies strongly locked to the Brillouin resonance significantly simplifies the traditional BOTDA setup, in particular, avoiding the use of broadband electrooptical modulators and related electronic circuits.

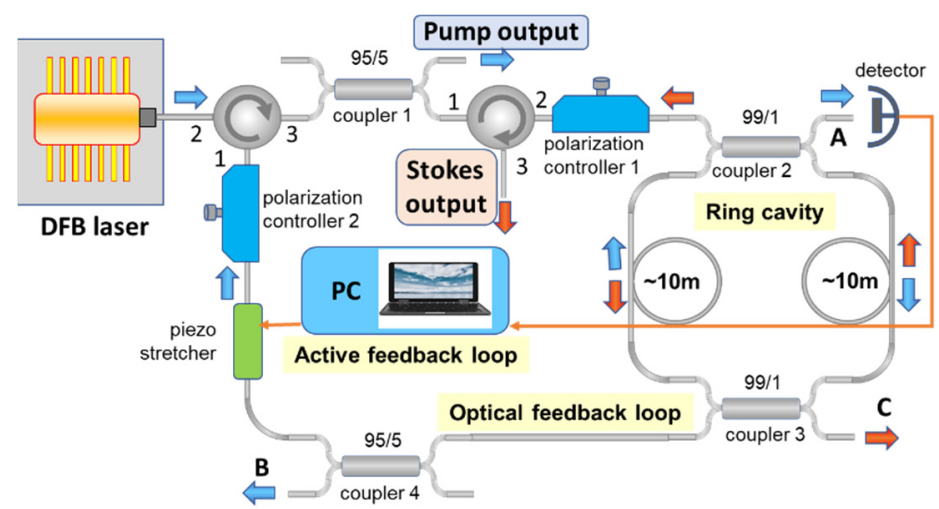

Figure A1. Dual-frequency laser sources used as the master-oscillator in BOTDA setup.

\section{Appendix A.1. Laser Configuration and Principle of Operation \\ Appendix A.1.1. Experimental Laser Configuration}

The experimental configuration of the dual-frequency laser is shown in Figure A1. The semiconductor laser was a commercial distributed feedback (DFB) laser diode (MITSUBISHI FU-68PDF-V520M27B, Mitsubishi Electric Corporation) delivering 15 mW at $\sim 1535 \mathrm{~nm}$. It is assembled within a standard 14-pin butterfly package. The DFB laser is coupled to the fiber ring cavity through a circulator. The high-Q external ring cavity is spliced from two (99/1) couplers. Totally, it comprises a $20 \mathrm{~m}$ length of SMS-28 fiber (Corning, Inc.) perfectly adjusted for lasing at the Stokes Brillouin frequency [37]. To implement the self-injection locking, the first coupler provides passive feedback to the laser operation, redirecting a part of the light circulating clockwise $(\mathrm{CW})$ inside the cavity through the circulator back into the DFB laser cavity. Polarization controller 1 is used to adjust the polarization state of the light inside the fiber ring cavity, enabling an optimal coupling between the laser radiation and one of the ring cavity modes. Polarization controller 2 adjusts the polarization state of the light before its injection into the DFB laser emitting a linear polarization, thus controlling the optical feedback strength. A piezo fiber-stretcher (PFS, Evanescent Optics Inc., Model 915B) is attached to the feedback loop. It is used as an optical phase shitter driven by a low-cost USB Multifunction DAQ (National Instrument NI USB-6009). The DAQ is connected with a PC and helps the laser maintain the desired operation in self-injection locking regime. The fiber configuration is spliced from standard 
telecom components and placed into a foam box stabilized at $\sim 25^{\circ} \mathrm{C}$, thus protecting the laser system from the laboratory environment. A fast photodetector detects the optical signal at port $\mathrm{A}$, which is used as an error signal for operation of the active feedback circuit. The active feedback circuit tends to keep it as low as possible, providing an appropriate voltage to the piezo fiber-stretcher. Additional thermal control is applied to the laser box as a whole and used to keep the voltage applied to the piezo-stretcher within its dynamic range.

\section{Appendix A.1.2. Operation and Stabilization Mechanisms}

The mechanism of the laser operation and stabilization is discussed in $[33,34,36,38]$. A simplified view on the laser operation is depicted in Figure A2. The light emitted by a DFB laser passes the fiber ring cavity and optical feedback loop and is injected back into the DFB laser cavity, forcing the DFB laser to emit at $v_{L}=v_{R}$, where $v_{R}$ is one of the ring cavity resonant frequencies. For stable laser operation, the laser frequency $v_{L}$ should also be resonant for the coupled cavity comprising the DFB laser cavity and feedback loop $v_{F B+L D}$, so $v_{L}=v_{R}=v_{L D+F B}$. The position of the frequency $v_{L D+F B}$ with respect to $v_{R}$ is controlled by the piezo-stretcher through the applied voltage. The laser operation becomes unstable when the environment noise affecting the laser configuration fibers violates the equality $v_{R}=v_{L D+F B}$. The task of the active feedback circuit is to maintain the equality $v_{R}=v_{L D+F B}$, minimizing the error signal detected at port A. In this case, the laser power at $v_{L}=v_{R}$ is perfectly coupled to the fiber ring cavity and enables effective backward lasing at Stokes frequency $v_{S}=v_{L}-\Delta_{S B S}$, where $\Delta_{S B S}$ is the Brillouin frequency shift. With the well-adjusted fiber ring cavity length, the Stokes frequency coincides with one of the resonant ring resonances. Therefore, the perfect stabilization of the lasing at $v_{L}=v_{R}=v_{L D+F B}$ ensures stabilization of lasing at frequency $v_{S}$.

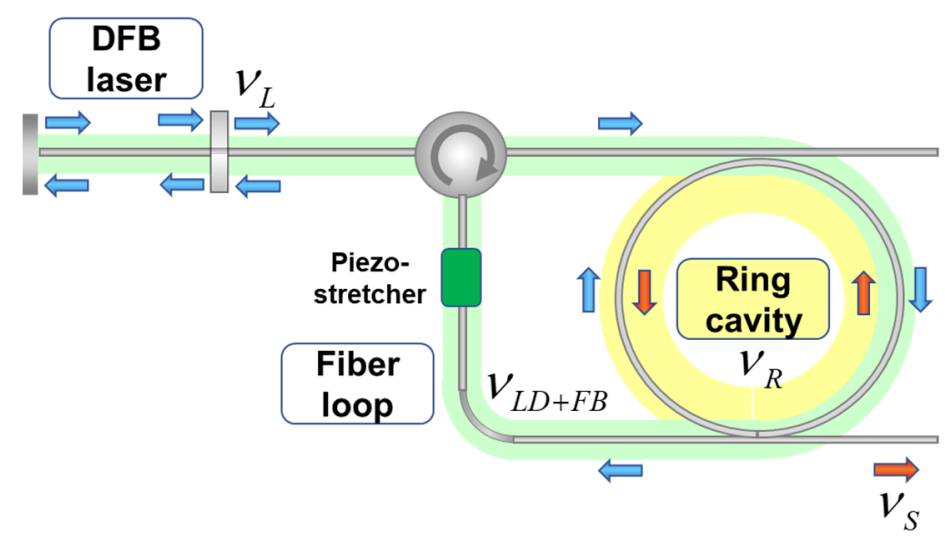

Figure A2. Illustration to dual-frequency laser operation. Green background shows the coupled cavity comprising the DFB laser and feedback loop; yellow background marks the ring cavity.

In accordance with our observations, there are two cases when mode-hopping in the laser occurs. First, a mechanical vibration such as a pencil knock on the laser box that causes short-time perturbations of the laser power (see Figure A3). We used a foam box to protect the laser from mechanical vibrations. Second is an extended temperature drift. While the active feedback circuit operates to counteract temperature drift, the voltage applied to the piezo-stretcher could exhaust its dynamic range ( $\sim 40 \mathrm{rad})$. In this case, for further operation, the phase delay induced by the piezo-stretcher should be reset by an integer number of $2 \pi$. Such a jump destabilizes the laser in the same way as the pencil kick. To avoid destabilization, the working point of the piezo-stretcher should be always maintained within its dynamic range. In our setup, this was implemented through the additional thermal control applied to the laser box and using the voltage applied to the piezo-stretcher as an error signal. 


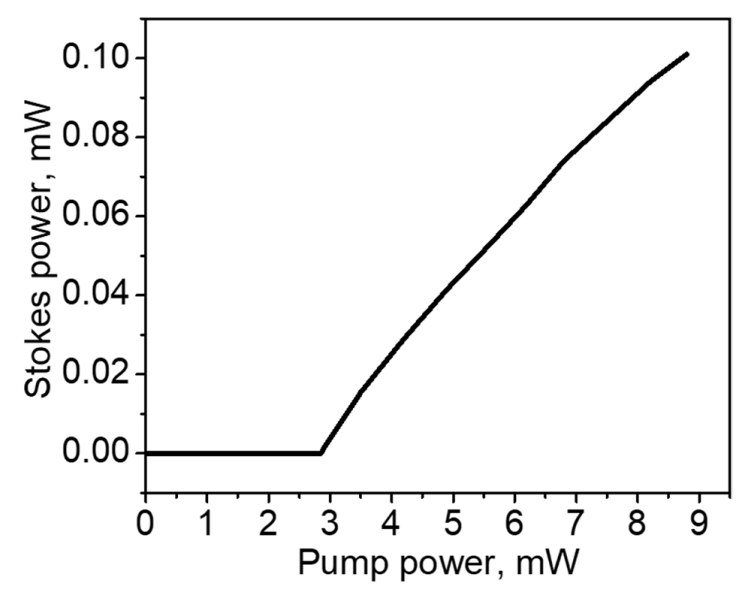

Figure A3. Stokes output power as a function of the pump output power.

\section{Appendix A.2. Laser Performance Characteristics}

Appendix A.2.1. Output Power

The maximal laser output powers emitted by the laser are $\sim 9 \mathrm{~mW}$ and $\sim 100 \mu \mathrm{W}$ for the pump and Stokes laser outputs shown in Figure A1, respectively. The experimental dependence of the laser power at the Stokes frequency (Stokes output) on the laser power at the pump frequency (pump output) is shown in Figure A3. The pump power threshold for the Stokes frequency generation is $\sim 2.9 \mathrm{~mW}$. The pump-to-Stokes power conversion efficiency above the threshold is $\sim 3.3 \%$.

\section{Appendix A.2.2. Laser Oscilloscope Traces and Stability against Perturbations}

The typical laser oscilloscope traces recorded at pump output, Stokes output, optical error signal (port A), and the electrical feedback signal are shown in Figure A4. The recorded traces provide insight into the system stability, demonstrating the system response to a pencil knock on the spliced fiber setup. Due to the active feedback circuit, the power at port A (used as the error signal) always tends to its minimal value, thus enabling stable laser operation at two frequencies. All unperturbed optical power traces are almost flat and exhibit no fluctuations. When the configuration was perturbed by the pencil knock, the traces exhibited dynamic behavior. The optical error signal makes a few stochastic fluctuations and then stabilizes to the original level. The time constants of the feedback mechanism are $\tau_{L} \sim 0.2 \mathrm{~s}$ and $\tau_{S} \sim 0.05 \mathrm{~s}$ for the pump and Stokes outputs, respectively.

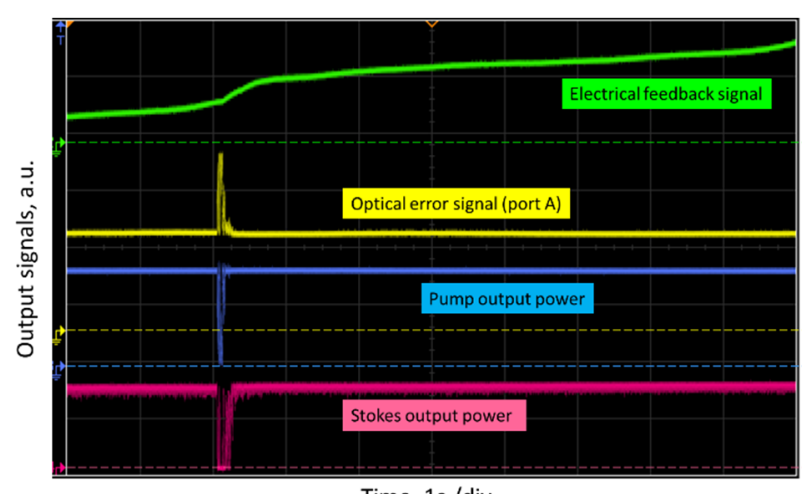

Figure A4. Typical oscilloscope traces of the optical error signal (port A), pump, and Stokes output powers and electrical feedback signal, highlighting the response to a pencil knock on the laser box. 


\section{Appendix A.2.3. Laser Polarization States}

The degree of polarization measured by the polarization analyzer (HP 8509A $\backslash \mathrm{B}$ ) are $\sim 100 \%$ for the pump and Stokes laser outputs.

\section{Appendix A.2.4. Lorentzian Laser Linewidths}

The self-heterodyne spectra $[39,40]$ of the pump and Stokes laser radiation averaged over 10 measurements each are shown in Figure A5. The measurements were performed with a fiber Mach-Zehnder interferometer (comprising the $55 \mathrm{~km}$ delay fiber and $25 \mathrm{MHz}$ phase modulator), $\sim 5 \mathrm{GHz}$ photodetector, and RF spectrum analyzer (FSH8, Rohde \& Schwarz). The recorded spectra were fitted by the Voigt profile and decomposed into the Gaussian and Lorentzian contributions [40,41]. The fitting parameter $w_{L}$ relates to the laser Lorentzian linewidth as $1 / 2 w_{L}$, resulting in the measured values of $\sim 270 \mathrm{~Hz}$ and $\sim 110 \mathrm{~Hz}$ for pump and Stokes laser outputs, respectively [40].

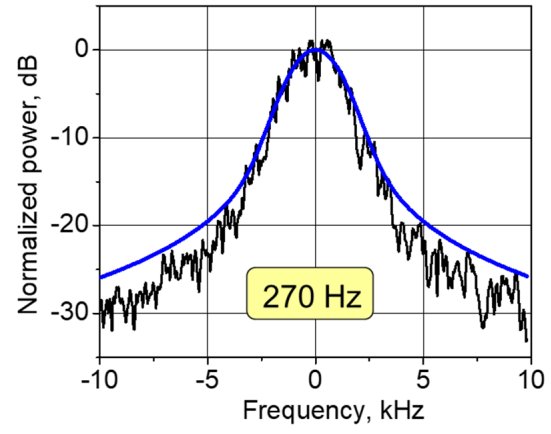

(a)

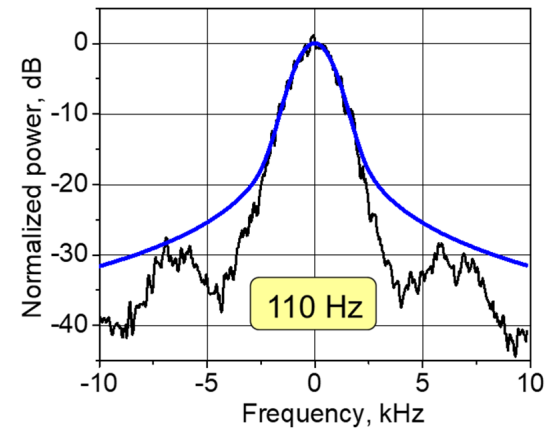

(b)

Figure A5. The self-heterodyne spectra of the pump (a) and Stokes (b) laser radiation: the measured spectra (black) and their fitting by the Voigt functions (blue). The fitting parameters evaluating the Gaussian and Lorentzian contributions are $w_{G}=2.2 \mathrm{kHz}, w_{L}=540 \mathrm{~Hz}$ and $w_{G}=1.7 \mathrm{kHz}$, $w_{L}=220 \mathrm{~Hz}$, for the pump and Stokes outputs, respectively.

Appendix A.2.5. Noise Performance

The power spectral density (PSD) of phase noise measured for the pump and Stokes radiations with an RF spectrum analyzer (Agilent N9320A) in the range of $10-100 \mathrm{kHz}$ is shown in Figure A6a. The measurements were performed by the self-heterodyne method [42-44] with the Mach-Zehnder interferometer comprising a $\sim 1.3 \mathrm{~km}$ delay fiber $(\sim 5.76 \mu \mathrm{s})$ and $20 \mathrm{MHz}$ frequency modulator. In comparison with the free-running laser, the measured PSD is lower by $\sim(35-40)$ and $\sim(50-60) \mathrm{dBc} / \mathrm{Hz}$ for the pump and Stokes laser outputs, respectively. The relative intensity noise (RIN) measured with a lock-in amplifier SRS510 in the range of 1-100 kHz is shown in Figure A6b. In comparison with the free-running laser, the measured RIN is lower by $\sim(5-10) d B$ and higher by $30-40 \mathrm{~dB}$ for the pump and Stokes laser outputs, respectively.

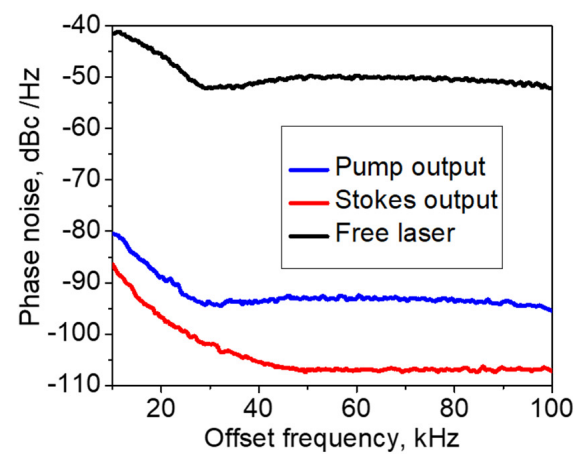

(a)

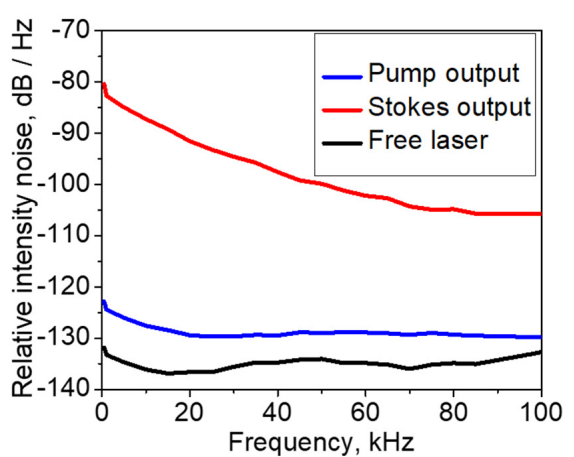

(b)

Figure A6. Noise performance of the laser: (a) phase noise; (b) relative intensity noise (RIN). 


\section{References}

1. Lee, B. Review of the present status of optical fiber sensors. Opt. Fiber Technol. 2003, 9, 57-79. [CrossRef]

2. Ahsani, V.; Ahmed, F.; Jun, M.B.; Bradley, C. Tapered fiber-optic Mach-Zehnder interferometer for ultra-high sensitivity measurement of refractive index. Sensors 2019, 19, 1652. [CrossRef]

3. Fotiadi, A.A.; Brambilla, G.; Ernst, T.; Slattery, S.A.; Nikogosyan, D.N. TPA-induced long-period gratings in a photonic crystal fiber: Inscription and temperature sensing properties. J. Opt. Soc. Am. B Opt. Phys. 2007, 24, 1475-1481. [CrossRef]

4. Zheng, H.; Zhang, J.; Guo, N.; Zhu, T. Distributed Optical Fiber Sensor for Dynamic Measurement. J. Lightwave Technol. 2021, 39, 3801-3811. [CrossRef]

5. Bao, X.; Zhou, Z.; Wang, Y. Review: Distributed time-domain sensors based on Brillouin scattering and FWM enhanced SBS for temperature, strain and acoustic wave detection. PhotoniX 2021, 2, 14. [CrossRef]

6. Faustov, A.V.; Gusarov, A.V.; Mégret, P.; Wuilpart, M.; Zhukov, A.V.; Novikov, S.G.; Svetukhin, V.V.; Fotiadi, A.A. Application of phosphate doped fibers for OFDR dosimetry. Results Phys. 2016, 6, 86-87. [CrossRef]

7. Ding, Z.; Wang, C.; Liu, K.; Jiang, J.; Yang, D.; Pan, G.; Pu, Z.; Liu, T. Distributed Optical Fiber Sensors Based on Optical Frequency Domain Reflectometry: A review. Sensors 2018, 18, 1072. [CrossRef]

8. Kurashima, T.; Horiguchi, T.; Tateda, M. Distributed-temperature sensing using stimulated Brillouin scattering in optical silica fibers. Opt. Lett. 1990, 15, 1038-1040. [CrossRef] [PubMed]

9. Fellay, A.; Thévenaz, L.; Facchini, M.; Niklès, M.; Robert, P. Distributed sensing using stimulated Brillouin scattering: Towards ultimate resolution. In Proceedings of the 12th International Conference on Optical Fiber Sensors, Williamsburg, VA, USA, 28 October 1997; p. OWD3.

10. Bao, X.; Webb, D.J.; Jackson, D.A. 22-km distributed temperature sensor using Brillouin gain in an optical fiber. Opt. Lett. 1993, 18, 552-554. [CrossRef] [PubMed]

11. Johny, J.; Amos, S.; Prabhu, R. Optical Fibre-Based Sensors for Oil and Gas Applications. Sensors 2021, 21, 6047. [CrossRef]

12. Hsu, W.-K.; Lee, Y.-L.; Kuan, T.-T. Brillouin Frequency Shift Sensing Technology Used in Railway Strain and Temperature Measurement. Appl. Sci. 2021, 11, 7101. [CrossRef]

13. Shen, J.; Li, T.; Zhu, H.; Yang, C.; Zhang, K. Sensing Properties of Fused Silica Single-Mode Optical Fibers Based on PPP-BOTDA in High-Temperature Fields. Sensors 2019, 19, 5021. [CrossRef]

14. Zhang, D.; Yang, Y.; Xu, J.; Ni, L.; Li, H. Structural Crack Detection Using DPP-BOTDA and Crack-Induced Features of the Brillouin Gain Spectrum. Sensors 2020, 20, 6947. [CrossRef] [PubMed]

15. Bado, M.F.; Casas, J.R. A Review of Recent Distributed Optical Fiber Sensors Applications for Civil Engineering Structural Health Monitoring. Sensors 2021, 21, 1818. [CrossRef]

16. Dong, Y. High-Performance Distributed Brillouin Optical Fiber Sensing. Photonic Sens. 2021, 11, 69-90. [CrossRef]

17. Soto, M.A. Distributed Brillouin Sensing: Time-Domain Techniques; Peng, G.D., Ed.; Springer: Singapore, 2018.

18. Wang, B.; Dong, Y.; Ba, D.; Bao, X. High spatial resolution: An integrative review of its developments on the Brillouin optical time- and correlation-domain analysis. Meas. Sci. Technol. 2020, 31, 052001. [CrossRef]

19. Dominguez-Lopez, A.; Soto, M.A.; Martin-Lopez, S.; Thevenaz, L.; Gonzalez-Herraez, M. Resolving 1 million sensing points in an optimized differential time-domain Brillouin sensor. Opt. Lett. 2017, 42, 1903-1906. [CrossRef] [PubMed]

20. Zhou, D.; Dong, Y.; Wang, B.; Pang, C.; Ba, D.; Zhang, H.; Lu, Z.; Li, H.; Bao, X. Single-shot BOTDA based on an optical chirp chain probe wave for distributed ultrafast measurement. Light Sci. Appl. 2018, 7, 32. [CrossRef]

21. Brown, A.W.; Smith, J.P.; Bao, X.; Demerchant, M.D.; Bremner, T. Brillouin Scattering Based Distributed Sensors for Structural Applications. J. Intell. Mater. Syst. Struct. 2016, 10, 340-349. [CrossRef]

22. Nikles, M.; Thevenaz, L.; Robert, P.A. Simple distributed fiber sensor based on Brillouin gain spectrum analysis. Opt. Lett. 1996, 21, 758. [CrossRef]

23. Iribas, H.; Urricelqui, J.; Mariñelarena, J.; Sagues, M.; Loayssa, A. Cost-Effective Brillouin Optical Time-Domain Analysis Sensor Using a Single Optical Source and Passive Optical Filtering. J. Sens. 2016, 2016, 8243269. [CrossRef]

24. Song, K.Y.; Yang, S. Simplified Brillouin optical time-domain sensor based on direct modulation of a laser diode. Opt. Express 2010, 18, 24012-24018. [CrossRef] [PubMed]

25. Bravo, M.; Ullan, A.; Zornoza, A.; Loayssa, A.; Lopez-Amo, M.; Lopez-Higuera, J.M. Application of remote power-by-light switching in a simplified BOTDA sensor network. Sensors 2013, 13, 17434-17444. [CrossRef] [PubMed]

26. Marini, D.; Iuliano, M.; Bastianini, F.; Bolognini, G. BOTDA Sensing Employing a Modified Brillouin Fiber Laser Probe Source. J. Lightwave Technol. 2018, 36, 1131-1137. [CrossRef]

27. Rossi, L.; Marini, D.; Bastianini, F.; Bolognini, G. Analysis of enhanced-performance fibre Brillouin ring laser for Brillouin sensing applications. Opt. Express 2019, 27, 29448-29459. [CrossRef] [PubMed]

28. Rossi, L.; Marini, D.; Bastianini, F.; Bolognini, G. Enhanced performance short cavity Brillouin fiber ring laser for high-stability BOTDA sensing. In Proceedings of the 2020 IEEE Sensors, San Jose, CA, USA, 25-28 October 2020; pp. 1-4.

29. Spirin, V.V.; Lopez-Mercado, C.A.; Mégret, P.; Korobko, D.A.; Zolotovskii, I.O.; Fotiadi, A.A. Stabilizing Brillouin fiber laser for applications in distributed BOTDA sensing. In Proceedings of the Optical Sensors 2021, Online Conference, 18 April 2021; p. 1177207.

30. Bueno Escobedo, J.L.; Spirin, V.V.; López-Mercado, C.A.; Mégret, P.; Zolotovskii, I.O.; Fotiadi, A.A. Self-injection locking of the DFB laser through an external ring fiber cavity: Polarization behavior. Results Phys. 2016, 6, 59-60. [CrossRef] 
31. Bueno Escobedo, J.L.; Spirin, V.V.; López-Mercado, C.A.; Márquez Lucero, A.; Mégret, P.; Zolotovskii, I.O.; Fotiadi, A.A. Selfinjection locking of the DFB laser through an external ring fiber cavity: Application for phase sensitive OTDR acoustic sensor. Results Phys. 2017, 7, 641-643. [CrossRef]

32. Bueno Escobedo, J.L.; Jason, J.; López-Mercado, C.A.; Spirin, V.V.; Wuilpart, M.; Mégret, P.; Korobko, D.A.; Zolotovskiy, I.O.; Fotiadi, A.A. Distributed measurements of vibration frequency using phase-OTDR with a DFB laser self-stabilized through PM fiber ring cavity. Results Phys. 2019, 12, 1840-1842. [CrossRef]

33. Spirin, V.V.; Bueno Escobedo, J.L.; Korobko, D.A.; Mégret, P.; Fotiadi, A.A. Dual-frequency laser comprising a single fiber ring cavity for self-injection locking of DFB laser diode and Brillouin lasing. Opt. Express 2020, 28, 37322-37333. [CrossRef]

34. Spirin, V.V.; Bueno Escobedo, J.L.; Korobko, D.A.; Mégret, P.; Fotiadi, A.A. Stabilizing DFB laser injection-locked to an external fiber-optic ring resonator. Opt. Express 2020, 28, 478-484. [CrossRef] [PubMed]

35. Stiller, B.; Kudlinski, A.; Lee, M.W.; Bouwmans, G.; Delque, M.; Beugnot, J.-C.; Maillotte, H.; Sylvestre, T. SBS Mitigation in a Microstructured Optical Fiber by Periodically Varying the Core Diameter. IEEE Photonics Technol. Lett. 2012, 24, 667-669. [CrossRef]

36. Spirin, V.V.; Bueno Escobedo, J.L.; Miridonov, S.V.; Maya Sánchez, M.C.; López-Mercado, C.A.; Korobko, D.A.; Zolotovskii, I.O.; Fotiadi, A.A. Sub-kilohertz Brillouin fiber laser with stabilized self-injection locked DFB pump laser. Opt. Laser Technol. 2021, 141, 107156. [CrossRef]

37. López-Mercado, C.A.; Spirin, V.V.; Kablukov, S.I.; Zlobina, E.A.; Zolotovskiy, I.O.; Mégret, P.; Fotiadi, A.A. Accuracy of single-cut adjustment technique for double resonant Brillouin fiber lasers. Opt. Fiber Technol. 2014, 20, 194-198. [CrossRef]

38. Korobko, D.A.; Zolotovskii, I.O.; Panajotov, K.; Spirin, V.V.; Fotiadi, A.A. Self-injection-locking linewidth narrowing in a semiconductor laser coupled to an external fiber-optic ring resonator. Opt. Commun. 2017, 405, 253-258. [CrossRef]

39. Derickson, D.; Hentschel, C.; Vobis, J. Fiber Optic Test and Measurement; Prentice Hall: Hoboken, NJ, USA, 1998 ; Volume 8.

40. Mercer, L.B. 1/f Frequency Noise Effects on Self-Heterodyne Linewidth Measurements. IEEE Lightwave Technol. 1991, 9, 485-493. [CrossRef]

41. Chen, M.; Meng, Z.; Wang, J.; Chen, W. Ultra-narrow linewidth measurement based on Voigt profile fitting. Opt. Express 2015, 23, 6803-6808. [CrossRef]

42. Camatel, S.; Ferrero, V. Narrow Linewidth CW Laser Phase Noise Characterization Methods for Coherent Transmission System Applications. J. Lightwave Technol. 2008, 26, 3048-3055. [CrossRef]

43. Llopis, O.; Merrer, P.H.; Brahimi, H.; Saleh, K.; Lacroix, P. Phase noise measurement of a narrow linewidth CW laser using delay line approaches. Opt. Lett. 2011, 36, 2713-2715. [CrossRef]

44. Li, Y.; Fu, Z.; Zhu, L.; Fang, J.; Zhu, H.; Zhong, J.; Xu, P.; Chen, X.; Wang, J.; Zhan, M. Laser frequency noise measurement using an envelope-ratio method based on a delayed self-heterodyne interferometer. Opt. Commun. 2019, 435, 244-250. [CrossRef] 